\title{
INSTRUMENTOS DE AVALIAÇÃO DO DESENVOLVIMENTO INFANTIL DE RECÉM-NASCIDOS PREMATUROS
}

\section{INSTRUMENTS OF EVALUATION OF CHILD DEVELOPMENT OF PREMATURE NEWBORNS}

Naíme Diane Sauaia Holanda Silva ${ }^{1}$

Fernando Lamy Filho ${ }^{2}$

Mônica Elinor Alves Gama ${ }^{3}$

Zeni de Carvalho Lamy ${ }^{4}$

André do Lago Pinheiro ${ }^{5}$

Diego do Nascimento Silva ${ }^{6}$

Silva NDSH et al. Instrumentos de avaliação do desenvolvimento infantil de recém-nascidos prematuros. Rev. Bras. Cresc. e Desenv. Hum. 2011; 21(1): 85-98.

\section{Resumo:}

A melhoria significativa dos cuidados intensivos neonatais causou uma redução na mortalidade em necém-nascidos de risco e, portanto, um aumento de sua morbidade, o que realçou a importância da avaliação do desenvolvimento dessas crianças. Esta revisão de literatura foi desenhada para identificar os principais instrumentos indicados para avaliar o desenvolvimento dos pré-termos. As bases de dados utilizadas foram Medline, Lilacs, Scielo, manuais dos instrumentos e livros na área de Pediatria por apresentarem maiores detalhes sobre os instrumentos e não estabelecer limite de tempo, pois o objetivo da pesquisa foi identificar desde os instrumentos mais antigos até os publicados recentemente. Foram identificados 11 instrumentos de avaliação do desenvolvimento publicados desde 1947 a. O conhecimento dessas escalas e dos testes pode ajudar aos profissionais de saúde e pesquisadores que trabalham com o desenvolvimento de crianças prematuras, pois constituem uma ferramenta adicional durante o processo de avaliação. A escolha do instrumento de avaliação deve ser realizada em acordo com as necessidades de cada criança. Podendo ser utilizados para a triagem, diagnóstico, planejamento e acompanhamento do tratamento.

Palavras-chave: prematuridade; desenvolvimento infantil; avaliação do desenvolvimento infantil.

1 Mestra, Programa de Pós - graduação em Saúde Materno-Infantil, Universidade Federal do Maranhão - UFMA , São Luís - MA. Professora, Centro Universitário do Maranhão (UNICEUMA), São Luís - MA.

2 Doutor, Instituto Fernandes Figueira, Fundação Oswaldo Cruz (Fiocruz), Rio de Janeiro, RJ. Médico Neonatologista pesquisador. Professor, Programa de Pós - graduação em Saúde Materno-Infantil, Universidade Federal do Maranhão UFMA , São Luís - MA.

3 Doutora, Médica Pediatra pesquisadora. Professora, Programa de Pós - graduação em Saúde Materno-Infantil, Universidade Federal do Maranhão - UFMA , São Luís - MA.

4 Doutora, Médica Neonatologista pesquisadora. Professora, Programa de Pós - graduação em Saúde Materno-Infantil, Universidade Federal do Maranhão - UFMA, São Luís - MA.

5 Bolsista Voluntário de Iniciação Científica CNPq/UFMA. Acadêmico de Medicina pela Universidade Federal do Maranhão UFMA, São Luís - MA.

6 Bolsista de Extensão pela Pró-Reitoria de Extensão da Universidade Federal do Maranhão - PROEX/UFMA. Acadêmico de Medicina pela Universidade Federal do Maranhão - UFMA, São Luís - MA

Correspondência para: Naíme Diane Sauaia Holanda Silva - Rua Primeiro de Maio, 242 Monte Castelo - CEP: 65035-520 -- São Luís, MA - E-mail: naimediane@hotmail.com - Fone: (98) 3221-2312 / (98) 9991-8224. 


\begin{abstract}
:
A significant improvement in neonatal intensive care has caused a reduction in mortality in babies at risk and therefore an increase in its morbidity, which stressed the importance of assessing the development of these children. This review was designed to identify the main instruments indicated to assess the development of preterm infants. The databases used were Medline, Lilacs, SciELO, manuals, tools and books in the field of Pediatrics for presenting details on the instruments and not set time limit, because the research objective was to identify the instruments from ancient to most published recently. We identified 11 instruments for assessing development published from 1947 to 1994. Knowledge of these scales and tests can help health professionals and researchers working on the development of premature children, since they constitute an additional tool in the evaluation process. The choice of instrument of assessment should be conducted in accordance with the needs of each child. Can be used for screening, diagnosis, treatment planning and monitoring.
\end{abstract}

Key words: prematurity; child development; assessment of child development.

\section{INTRODUÇÃO}

Os avanços tecnológicos das Unidades de Terapia Intensiva Neonatais (UTIN), incluindo a sofisticação de equipamentos, trabalho de humanização da equipe multidisciplinar e a inclusão da família no cuidado desses recémnascidos (RN), tem permitido maior sobrevida dos prematuros. Isso tem gerado preocupação em curto e longo prazos quanto ao desenvolvimento dessas crianças ${ }^{1}$. Esse aumento do número de recém-nascidos prematuros provocou um interesse maior dos pesquisadores em investigar o desenvolvimento neuropsicomotor dos mesmos ${ }^{2}$.

O acompanhamento do desenvolvimento neuropsicomotor do recém-nascido de risco, como os prematuros, e a investigação específica de suas condições clínicas traduzem-se especificamente em uma atuação preventiva por meio da detecção precoce de alguma anormalidade e apropriado encaminhamento para tratamento específico ${ }^{2,3}$.
De acordo com o que foi exposto anteriormente, percebe-se que o diagnóstico precoce continua sendo um desafio para o profissional da saúde, na avaliação e compreensão precisas do significado de qualquer atraso e dos limites da normalidade. A intervenção precoce no desenvolvimento motor do RN prematuro passa a ser um importante instrumento para estimular a criança a desenvolver suas capacidades de forma plena, limitando sinais de sequelas e invalidez ${ }^{4}$.

A avaliação do desenvolvimento motor faz parte do protocolo de seguimento de egressos das UTIN, e inúmeras ferramentas têm sido utilizadas para auxiliar os profissionais da área a fazerem uma avaliação funcional mais precisa ${ }^{5}$.

Na avaliação do desenvolvimento neuropsicomotor, deve-se destacar a importância do uso de escalas confiáveis, de comprovada sensibilidade e especificidade, e que representem a diversidade cultural dos indivíduos ${ }^{6}$. 
Existem vários instrumentos padronizados que auxiliam na identificação dessas crianças de risco e muitos deles são utilizados em estudos, para verificar sua eficácia ou seu valor preditivo, ou na prática clínica dentro dos programas de follow-up*. Esses testes e escalas de desenvolvimento facilitam e auxiliam tanto a triagem e o diagnóstico quanto o planejamento e progressão do tratamento, caso alguma anormalidade seja detectada ${ }^{7,8}$.

Portanto, o profissional deve estar familiarizado com os diversos instrumentos de avaliação existentes para, assim, selecionar o mais adequado na realização do seu serviço ou pesquisa. No Brasil, o desafio do diagnóstico de alterações motoras é agravado pela escassez de instrumentos de avaliação padronizados e validados para essa população, necessitando da utilização de testes e escalas internacionais que, na maioria dos casos, possuem manuais e protocolos publicados em língua inglesa ${ }^{8}$.

Desta maneira, o objetivo é descrever sobre os principais instrumentos de avaliação do desenvolvimento infantil aplicáveis aos recém-nascidos prematuros.

\section{MÉTODO}

A pesquisa foi realizada nas bases de dados Medline, Lilacs, Scielo, Plataforma CAPES, PubMed e Google Scholar utilizando-se as seguintes palavras-chave em português e inglês: desenvolvimento neuropsicomotor and instrumentos de avaliação do desenvolvimento and programas de acompanhamento and prematuridade.

Foram incluídos artigos sobre avaliação do desenvolvimento neuropsicomotor de prematuros, publicados em inglês e português. Foram excluídos artigos de intervenção, revi- são e trabalhos que não incluíram recém-nascidos prematuros. De forma complementar, livros das áreas de Neonatalogia e Pediatria, além de manuais sobre os principais instrumentos de avaliação do desenvolvimento da criança foram utilizados. Os manuais foram incluídos por apresentar maiores detalhes sobre os instrumentos. Não foi estabelecido limite de tempo, pois o objetivo da pesquisa foi identificar desde os instrumentos mais antigos até os publicados recentemente.

As informações coletadas foram organizadas de acordo com a ordem cronológica de publicação dos instrumentos. Na descrição de cada instrumento foram levantadas as seguintes informações: objetivo do teste ou escala, comportamentos ou itens avaliados, faixa etária específica avaliada, propriedades psicométricas e limitações quanto à aplicação do instrumento.

\section{RESULTADOS}

Foram identificados na revisão 11 instrumentos de avaliação do desenvolvimento neuropsicomotor indicados para avaliação e acompanhamento de prematuros, conforme Tabela 1.

\section{DISCUSSÃO}

Com base no levantamento dos principais instrumentos de avaliação verifica-se a existência de 11 tipos de instrumentos de avaliação do desenvolvimento de crianças de zero a dois anos de idade. Quanto à data de publicação, o teste mais antigo foi publicado em 1947 (Teste de Gesell) e o mais recente publicado

\footnotetext{
Follow-up: originado do inglês ‘follow-up' são programas de acompanhamento do crescimento e desenvolvimento do RN de risco originado na década de 80 por pediatras e neonatologistas
} 
Tabela 1: Instrumentos de avaliação do desenvolvimento neuropsicomotor de recém-nascidos prematuros.

\begin{tabular}{|c|c|c|c|}
\hline Tipo de teste & $\begin{array}{l}\text { Autor / Ano de } \\
\text { publicação }\end{array}$ & Faixa Etária & Descrição do Teste \\
\hline Teste de Gesell & $\begin{array}{c}\text { Arnold Gesell e } \\
\text { colaboradores/ } \\
1947\end{array}$ & $\begin{array}{l}4 \text { semanas - } \\
36 \text { meses }\end{array}$ & $\begin{array}{l}\text { - Corresponde a uma avaliação direta e a obser- } \\
\text { vação da qualidade e da integração de compor- } \\
\text { tamentos. } \\
\text { - As categorias de análise desta escala referem- } \\
\text { se às áreas: comportamento adaptativo; com- } \\
\text { portamento motor grosseiro e delicado; com- } \\
\text { portamento de linguagem e comportamento } \\
\text { pessoal-social. } \\
\text { - Constitui um bom instrumento de diagnósti- } \\
\text { co, porém com limitações importantes. }\end{array}$ \\
\hline
\end{tabular}

\begin{tabular}{|c|c|c|c|}
\hline $\begin{array}{c}\text { Escala de } \\
\text { Desenvolvimento } \\
\text { Infantil de Bayley } \\
\text { (BSID) }\end{array}$ & $\begin{array}{c}\text { Nancy Bayley/ } \\
1969\end{array}$ & 1 - 42 meses & $\begin{array}{l}\text { - Teste padronizado e referenciado do desen- } \\
\text { volvimento motor, cognitivo, de linguagem e } \\
\text { comportamental. } \\
\text { - Utilizado na intervenção precoce e na pesqui- } \\
\text { sa clínica. } \\
\text { - Necessita de treinamento específico para sua } \\
\text { utilização. }\end{array}$ \\
\hline Teste Denver & $\begin{array}{c}\text { William } \\
\text { Frankenburg e } \\
\text { Josiah Dodds/ } \\
1967\end{array}$ & $\begin{array}{l}1 \text { semana - } \\
61 / 2 \text { anos }\end{array}$ & $\begin{array}{l}\text { - Teste padronizado e referenciado do desen- } \\
\text { volvimento para aspectos: pessoal-social; mo- } \\
\text { tor-fino adaptativo; linguagem; motor grossei- } \\
\text { ro e comportamental. } \\
\text { - Utilizado para diagnosticar atraso no desen- } \\
\text { volvimento, embora com fraca especificidade e } \\
\text { viés populacional. }\end{array}$ \\
\hline
\end{tabular}

\begin{tabular}{ccc}
\hline Teste de Triagem & Milani & $0-2$ anos \\
Sobre o & Comparetti e & \\
Desenvolvimento & Gidoni/1967 & \\
de & \\
Milani-Comparetti & &
\end{tabular}

- Teste de investigação padronizado de comportamentos motores espontâneos, incluindo locomoção; sentar e ficar de pé.

- Inclui também respostas evocadas que correspondem reações de equilíbrio, protetoras, de endireitamento e reflexos primitivos.

- Útil para descrever o desenvolvimento de um RN baseado na integração de reflexos primitivos para o desenvolvimento do controle postural.

\begin{tabular}{|c|c|c|c|}
\hline $\begin{array}{c}\text { Gráfico do } \\
\text { Desenvolvimento } \\
\text { Motor de } \\
\text { Zdanska - Brincken }\end{array}$ & $\begin{array}{c}\text { Zdanska- } \\
\text { Brincken e } \\
\text { Wolanski/1969 }\end{array}$ & $\begin{array}{c}4 \text { semanas a } 1 \\
\text { ano }\end{array}$ & $\begin{array}{l}\text { - O teste tem como objetivo avaliar o controle } \\
\text { postural de crianças no } 1^{\circ} \text { ano de vida como } \\
\text { subsídio para o monitoramento de seu desen- } \\
\text { volvimento. } \\
\text { - Instrumento delineia quatro grids para deta- } \\
\text { lhar as mudanças do desenvolvimento a respei- } \\
\text { to de: movimentos da cabeça e do tronco, pos- } \\
\text { tura sentada, postura bípede e locomoção. }\end{array}$ \\
\hline
\end{tabular}


Tabela 1 (cont.): Instrumentos de avaliação do desenvolvimento neuropsicomotor de recémnascidos prematuros.

\begin{tabular}{|c|c|c|c|}
\hline Tipo de teste & $\begin{array}{c}\text { Autor / Ano de } \\
\text { publicação }\end{array}$ & Faixa Etária & Descrição do Teste \\
\hline $\begin{array}{c}\text { Escala de } \\
\text { Avaliação do } \\
\text { Comportamento do } \\
\text { Neonato (NBAS) }\end{array}$ & $\begin{array}{c}\text { T. Berry } \\
\text { Brazelton e J. } \\
\text { Kelvi Nugent/ } \\
1973\end{array}$ & $\begin{array}{l}\text { Pré-termo } \\
\text { com 37-48 } \\
\text { semanas } \\
\text { pós- } \\
\text { concepção }\end{array}$ & $\begin{array}{l}\text { - Teste baseado em critérios de habituação, res- } \\
\text { posta oromotora, função vestibular e de tronco, } \\
\text { comportamentos e interação social. } \\
\text { - Fornece informações sobre os padrões } \\
\text { interativos das crianças que podem ser utiliza- } \\
\text { dos para orientar pais e cuidadores. }\end{array}$ \\
\hline $\begin{array}{l}\text { Avaliação dos } \\
\text { Movimentos da } \\
\text { Criança (MAI) }\end{array}$ & $\begin{array}{l}\text { Lynnette } \\
\text { Chandler, Mary } \\
\text { Andrews e } \\
\text { Marcia Sanson/ } \\
1980\end{array}$ & $0-12$ meses & $\begin{array}{l}\text { - Teste baseado em critérios para tônus muscu- } \\
\text { lar, reflexos, reações automáticas e movimento } \\
\text { voluntário. } \\
\text { - O teste é baseado num cálculo de valor de ris- } \\
\text { co para identificação de RN com disfunção } \\
\text { motora. } \\
\text { - Apresentando melhores perfis de risco entre } \\
\text { 4-8 meses. }\end{array}$ \\
\hline $\begin{array}{c}\text { Avaliação } \\
\text { Neurológica de } \\
\text { Bebês Prematuros } \\
\text { e a Termo }\end{array}$ & $\begin{array}{c}\text { Lilly Dubowitz e } \\
\text { Victor Dubowitz/ } \\
1981\end{array}$ & $\begin{array}{l}\text { A termo até } 3 \\
\text { dias de vida e } \\
\text { pré-termo } \\
\text { estáveis }\end{array}$ & $\begin{array}{l}\text { - Teste baseado em critérios de maturação neu- } \\
\text { rológica. } \\
\text { - Testes de habituação, movimento, tônus, re- } \\
\text { flexos e respostas comportamentais. } \\
\text { - Testagem clássica para determinar maturação } \\
\text { e desvios neurológicos de bebê. } \\
\text { Apresentam limitados dados psicométricos. }\end{array}$ \\
\hline $\begin{array}{c}\text { Peabody } \\
\text { Developmental } \\
\text { Motor Scale } \\
\text { (Escala PDMS) }\end{array}$ & $\begin{array}{l}\text { Rhonda Folio e } \\
\text { Rebecca Fewell/ } \\
1982\end{array}$ & 1-72 meses & $\begin{array}{l}\text { - Teste padronizado e referenciado para avalia- } \\
\text { ção das habilidades motoras grosseiras e finas } \\
\text { divididas em seis subtestes: reflexos, estática, } \\
\text { locomoção, manipulação de objetos, garra e in- } \\
\text { tegração visuomotora. } \\
\text { - Muito útil em programas de intervenção pre- } \\
\text { coce para determinar elegibilidade do atendi- } \\
\text { mento. }\end{array}$ \\
\hline $\begin{array}{l}\text { TIMP (Test of } \\
\text { Infant Motor } \\
\text { Performance) }\end{array}$ & $\begin{array}{c}\text { Suzann } \\
\text { Campbell/1993 }\end{array}$ & $\begin{array}{l}\text { Pré-termo } \\
\text { nascidos com } \\
34 \text { semanas. } \\
\text { Pós- } \\
\text { concepção } \\
4 \text { meses após } \\
\text { o nascimento }\end{array}$ & $\begin{array}{l}\text { - Avalia movimentos funcionais da cabeça e con- } \\
\text { trole do tronco nas posições em prono, em } \\
\text { supino e de pé, com } 28 \text { itens avaliados em } \\
\text { dicotomia e } 31 \text { itens escalonados. } \\
\text { - Identifica RN com risco de resultado motor } \\
\text { pobre. } \\
\text { - Teste muito sensível para avaliar efeitos de } \\
\text { tratamento. }\end{array}$ \\
\hline $\begin{array}{l}\text { Alberta Infant } \\
\text { Motor Scale } \\
\text { (AIMS) }\end{array}$ & $\begin{array}{c}\text { Martha Piper e } \\
\text { Johanna Darrah/ } \\
1994\end{array}$ & 0 - 18 meses & $\begin{array}{l}\text {-Teste padronizado na observação das habilida- } \\
\text { des motoras grosseiras em quatro posições: em } \\
\text { prono, em supino, sentada e de pé. } \\
\text { - Identifica RN com atraso motor e avalia a } \\
\text { maturação da habilidade motora grosseira. }\end{array}$ \\
\hline
\end{tabular}


em 1994 (Escala Motora de Alberta). A descrição de cada um dos instrumentos encontrados na pesquisa é apresentada a seguir.

\subsection{TESTE DE GESELL}

Arnold Gesell e colaboradores, na década de 20, elaboraram um programa de testes com a finalidade de avaliar o comportamento da criança durante o desenvolvimento e assim realizar o exame diagnóstico de seus desvios. O teste sofreu modificações e atualizações, sendo conhecido atualmente como Escala de Desenvolvimento de Gesell e Amatruda ${ }^{9}$.

O teste de Gesell é um teste de referência, envolvendo a avaliação direta e a observação da qualidade e da integração de comportamentos ${ }^{10}$. Pode ser aplicado em crianças de quatro semanas até 36 meses de idade cronológica. As categorias de análise dessa escala referem-se às seguintes áreas: comportamento adaptativo (organização e adaptação sensório-motora, cognição); comportamento motor grosseiro e delicado (sustentação da cabeça, sentar, engatinhar, andar, manipulação de objetos com as mãos); comportamento de linguagem (expressiva ou receptiva); comportamento pessoal-social (relação com o meio-ambiente) $)^{9,11}$.

Os comportamentos acima descritos são observados nas idades-chave de: quatro semanas, 16 semanas, 28 semanas, 40 semanas, 12 meses, 18 meses, 24 meses e 36 meses. O resultado final é quantitativo e expresso como quociente de desenvolvimento (QD), quando a criança é prematura deve ser usada a idade corrigida na avaliação. Tais dados são comparados a uma escala elaborada a partir dos comportamentos padrão apresentados por crianças em determinadas faixas etárias ${ }^{9,12}$.

De acordo com Gesell e Amatruda ${ }^{9}$, a confiabilidade e validade desse teste são consideradas boas, constituindo-se em um bom instrumento diagnóstico, muito utilizado em pesquisas e em centros de reabilitação. Entre- tanto, o teste apresenta algumas limitações: não considera a movimentação espontânea do RN, sua qualidade de movimentos e baseia-se na teoria neuromaturacional do desenvolvimento.

\subsection{ESCALA DE DESENVOLVIMENTO INFANTIL DE BAYLEY (BSID)}

Originalmente publicadas em 1933, as escalas Bayley são consideradas possuidoras de propriedades psicométricas para avaliação do desenvolvimento infantil, sendo padronizada com referências normativas para crianças pequenas ${ }^{13}$.

As Escalas de Desenvolvimento Infantil de Bayley foram desenvolvidas por Nancy Bayley e colaboradores em 1933, sendo uma revisão das publicações de Bayley em $1969^{13}$. É um meio abrangente de avaliar o atual estado de desenvolvimento de uma criança em estado particular. Representam o resultado de mais de 40 anos de pesquisa e prática clínica com crianças pequenas. $\mathrm{O}$ teste foi padronizado em uma amostra de 1262 crianças americanas com idade de dois a 30 meses, divididas em 14 grupos, no ano de 1960. Apresenta três versões: BSID I publicada em 1969; BSID II publicada em 1983 e a mais atualizada BSID III publicada em $2006^{14}$.

As escalas BSID II estão reconhecidas entre as melhores escalas existentes na área de avaliação do desenvolvimento infantil, fornecendo resultados confiáveis, válidos e precisos do estado de desenvolvimento da criança em teste. Sua utilização como instrumento de pesquisa tem recebido grande suporte da comunidade científica, porém seu elevado custo e treino exigido para correta administração explicam porque sua utilização é quase sempre exclusiva de especialistas que trabalham com crianças pequenas ${ }^{15}$.

BSID-III corresponde a Escala de Bayley - III e ao Teste de Triagem Bayley-III (Screeningtest). Ao ser aplicado está subdividido em cinco domínios: Cognição, Linguagem (comunicação expressiva e receptiva), Motor 
(grosso e fino), Social-emocional e Componente adaptativo. Os três primeiros domínios são observados com a criança em situação de teste e os dois últimos são observados por meio de questionários preenchidos pelos pais ou cuidadores ${ }^{16}$.

A Escala de Bayley - III é uma atualização dos dados normativos da BSID-II com amostra contemporânea e representativa, indicado para avaliar crianças de um a 42 meses de idade, apresentando melhora do conteúdo dos testes, melhora da qualidade psicométrica e, consequentemente, maior utilidade clínica. Dentre estes domínios está a Escala Cognitiva que determina como a criança pensa, reage e aprende sobre o mundo ao seu redor e está composta de 91 itens; a Escala de Linguagem está subdividida em dois subtipos: Comunicação Receptiva - parte que determina como a criança reorganiza sons e como a criança entende, fala e direcionam palavras, composta de 49 itens - e a Comunicação Expressiva - parte que determina como a criança se comunica usando sons, gestos e palavras, composta de 48 itens $^{16}$.

A Escala Motora está subdividida em Escala Motora Grossa e Fina. A Escala Motora Grossa determina como a criança movimenta seu corpo em relação à gravidade, composta de 72 itens, e a Escala Motora Fina determina como a criança usa suas mãos e dedos para fazer algo, composta de 66 itens $^{16}$.

A utilização dessa escala no Brasil é possível, porém, com limitações, pois ainda não se dispõe de validação para nossa população e cultura ${ }^{17}$. Outras limitações importantes são: a escala só pode ser aplicada por profissionais especializados e treinados e o examinador deve ser cauteloso na aplicação da mesma em RN pré-termo ${ }^{18}$.

\subsection{TESTE DENVER}

Foi desenvolvido por Frankenburg e Dodds $^{19}$, em 1967, com o objetivo de direcionar o cuidado dos adultos para as crianças com riscos e não de diagnosticar atrasos no desen- volvimento ${ }^{20}$. Seu uso foi difundido em muitos países, sofrendo adaptações, o que incitou uma revisão e repadronização do teste, resultando no Teste Denver II em 1992²1.

O teste pode ser aplicado por vários profissionais da saúde em crianças de zero a seis anos, classificando-a dicotomicamente em risco ou normal. Composto por 125 itens distribuídos na avaliação de quatro áreas distintas do desenvolvimento neuropsicomotor: motricidade ampla, motricidade fina-adaptativa, comportamento pessoal-social e linguagem $^{22,23}$. Esses itens são registrados por meio da observação direta da criança e, para alguns deles, solicita-se que a mãe informe se o filho realiza ou não determinada tarefa. O teste Denver II apresenta bons índices de validade e confiabilidade (0,99 interobservador e 0,9 em teste reteste) e, portanto, largamente utilizado tanto em pesquisas quanto na prática clínica ${ }^{24}$.

O teste é considerado de fácil execução e oferece um manual para treinamento e orientações quanto a sua utilização. Pode ser aplicado por vários profissionais da saúde e, por isso, é um dos testes mais utilizados na triagem de atrasos, inclusive em nosso país. Embora o teste não seja validado para nossa população, uma adaptação cultural, não formal, foi realizada a fim de facilitar a sua aplicação ${ }^{24}$.

Outra vantagem é a larga faixa etária que o teste atinge, possibilitando o acompanhamento prolongado do desenvolvimento infantil. Esta nova versão do teste também foi normatizada e validada cuidadosamente para a população do Colorado, EUA, e parece demonstrar maior sensibilidade na identificação de atraso em relação à primeira versão, especialmente na área da linguagem ${ }^{23,24}$.

Uma das desvantagens ressaltadas pelos pesquisadores é que, como o teste não foi criado para diagnosticar atrasos, mas para direcionar os cuidados com a criança, ele oferece resultados com pouco valor prognóstico, especialmente nos casos em que o número de respostas falhas é pequeno ${ }^{24}$. 
Apesar de o teste abranger uma larga faixa etária e permitir o acompanhamento longitudinal do desenvolvimento, parece ser insuficiente para avaliar mudanças qualitativas ao longo do tempo e detectar precocemente alterações psicomotoras sutis ${ }^{24}$.

\subsection{TESTE DE TRIAGEM SOBRE O DESENVOLVIMENTO DE MILANI- COMPARETTI}

O teste foi desenvolvido em 1967 pelos neurologistas italianos Milani Comparetti e Gidoni, avalia o desenvolvimento motor com base na correlação entre as aquisições funcionais motoras da criança e as estruturas reflexas. O instrumento é indicado para crianças a partir do nascimento até dois anos de idade. A forma de pontuação original sofreu modificações feitas pelos profissionais do Instituto de Reabilitação de Crianças Meyer, no Centro Médico da Universidade de Nebraska - EUA, em $1992^{25}$.

O tempo para administrar esse exame de triagem é de quatro a oito minutos. Observamse tanto comportamentos espontâneos (controle postural e padrões de movimentos ativos) quanto respostas evocadas (reflexos primitivos, reações de endireitamento e equilíbrio) ${ }^{25,26}$.

A confiabilidade interobservador mostra uma porcentagem de concordância de 89 a $95 \%$ e a do teste reteste de 82 a 100\%, porém a validade preditiva do teste Milani-Comparetti não foi bem estabelecida ${ }^{26}$.

A construção do teste depende de observações objetivas além de declarações dos pais, o que constitui em alguns casos prejuízo quanto à fidedignidade das informações. Tal avaliação apresenta melhores resultados quando consideradas alterações motoras, havendo outras avaliações mais completas para a identificação de alterações neurológicas ${ }^{25}$.

As vantagens do teste ressaltadas pelos pesquisadores é que o mesmo é considerado prático, útil, de fácil aplicação, aprendizagem e pontuação, não necessitando de local ou equipamentos especiais ${ }^{25,27}$.

\subsection{GRÁFICO DO DESENVOLVIMEN- TO MOTOR DE ZDANSKA - BRINCKEN}

Em 1969, Maria Zdanska-Brincken e Napoleon Wolanski, publicaram na Polônia artigo que teve como objetivo avaliar o controle postural dos lactentes no primeiro ano de vida, como subsídio para o monitoramento do desenvolvimento da criança. Nesse trabalho os autores propuseram um gráfico a partir de um estudo longitudinal, de 1960 a 1964, com 212 crianças avaliadas de quatro semanas de idade até aquisição da marcha ${ }^{25,27}$.

Quatro grids foram criados para detalhar as mudanças do desenvolvimento a respeito de: movimentos da cabeça e do tronco, postura sentada, postura bípede e locomoção ${ }^{28}$.

Finalmente, foi construído o gráfico do desenvolvimento motor, que tem como objetivos principais: obter o percentual de crianças que adquirem certos comportamentos; avaliar não só o desenvolvimento de acordo com a idade, mas o grau de aceleração ou atraso; obter um gráfico final da representação total da avaliação nas áreas analisadas; e identificar diferenças entre desenvolvimento motor e físico ${ }^{28}$.

\subsection{ESCALA DE AVALIAÇÃO DO COMPORTAMENTO DO NEONATO (NBAS)}

A Escala de Avaliação do Comportamento do Neonato foi desenvolvida originalmente por Brazelton e colaboradores ${ }^{29}$ em 1973. É um instrumento de análise do comportamento neuromotor, desenvolvido para distinguir diferenças individuais entre RN sadios, especialmente as relacionadas ao comportamento social interativo. 
De acordo com Tecklin ${ }^{25}$, a NBAS é apropriada para testes em recém-nascidos de três dias até um mês de idade, tendo sido usada para estudar RN a termo e prematuros próximos ao termo (mínimo de 36 semanas de gestação), assim como aqueles de diferentes nacionalidades e etnias.

O exame consiste em avaliar, analisar e graduar 28 itens comportamentais (capacidade interativa, comportamento motor, organização do estado comportamental e organização fisiológica) e 18 itens de reflexos, delineando o estado comportamental da criança ${ }^{18,25}$.

A NBAS é indicada para avaliar problemas neurológicos, assim como um instrumento efetivo de ensino aos pais sobre o manejo da criança. Consiste em uma técnica valiosa que serve para diferenciar características comportamentais de recém-nascidos normais com propósitos clínicos e de pesquisa. No entanto, recomenda-se seu uso em crianças brancas cujas mães tiveram um parto sem muitas complicações e, quando aplicada em pré-termo, exigem-se critérios especiais e adaptações ${ }^{18}$.

A principal desvantagem do teste é a necessidade de um programa de treinamento específico para realizar o teste de forma confiável, isso implica custo financeiro e disponibilidade de tempo ${ }^{27}$.

\subsection{AVALIAÇÃO DOS MOVIMENTOS DA CRIANÇA (MAI)}

A Avaliação dos Movimentos da Criança é um teste muito utilizado por terapeutas nos EUA. Criado por Chandler e outros dois fisioterapeutas pediátricos, em 1980, o teste foi embasado na experiência clínica e na revisão literária do desenvolvimento normal de crianças, destinando-se a avaliar o desenvolvimento motor em crianças de até um ano que demonstram alto risco para distúrbios motores, a fim de contribuir para estabelecer as bases para intervenção precoce ${ }^{30,31}$.
O teste também foi criado para acompanhar os efeitos da fisioterapia, bem como fornecer suporte para pesquisa como instrumento de avaliação ${ }^{32,31}$. O teste exige habilidade específica do examinador e intenso manuseio da criança. O exame consiste na avaliação do tônus, reflexos primitivos, reações automáticas de endireitamento, equilíbrio e proteção, além de movimentos voluntários, obtidos por estímulos visuais e auditivos ou por meio da manifestação de marcos motores ${ }^{32,33}$.

Com a orientação de um treinamento acurado para a aplicação, foi destinado a ser utilizado por fisioterapeutas e terapeutas ocupacionais que atuam na área pediátrica, mas pode ser aplicado por diversos profissionais de saúde com experiência em desenvolvimento infantil ${ }^{32,33}$. Cada escore questionável constitui um ponto de risco para classificar a criança como normal ou questionável. Estes somados oferecem, ao final, um critério de risco total; quanto maior a pontuação evidenciada pela criança, maior o risco para o desenvolvimento ${ }^{33}$.

Embora os autores do teste não tenham desenvolvido uma escala normativa, existem perfis de referência para quatro, seis, oito e doze meses de idade ${ }^{32}$.

O teste é composto por 65 itens distribuídos em quatro aspectos: tônus, reflexos primitivos, reações automáticas, movimentos voluntários eliciados. O teste não tem escore normativo, mas foram criados perfis de desempenho para quatro, seis e oito meses ${ }^{33}$.

A confiabilidade interobservador determinada pelos autores foi maior que $90 \%$, consideramos uma das principais vantagens do teste a sua forma de abrangência, pois é um dos poucos testes que determina a qualidade do movimento. Tendo como principal desvantagem o extenso tempo de aplicação e manuseio com a criança, alguns itens apresentam confiabilidade questionável, necessitando ainda de estudos de validade para melhorar a qualidade do teste ${ }^{25,27}$. 


\subsection{AVALIAÇÃO NEUROLÓGICA DE RECÉM-NASCIDOS PREMATUROS E A TERMO}

Avaliação neurológica de recém-nascidos prematuros e a termo é uma avaliação neurológica e neurocomportamental formulada e elaborada por Dubowitz e Dubowitz ${ }^{34}$, em 1981. Consiste em um exame sistemático e rapidamente administrado (10 a 15 minutos), tanto para prematuros como para RN a termo, com a finalidade de detectar precocemente anormalidades neurológicas ${ }^{18}$.

Pode ser aplicado em crianças de zero a 12 meses, pois os criadores não possuem dados de acompanhamento por um tempo de mais de um ano. Sendo que o teste é composto de nove itens de neurocomportamento (capacidade do RN se habituar a estímulos luminosos e sonoros repetidos, movimentos espontâneos do corpo, reação defensiva, observação de movimentos oculares anormais; orientação auditiva e visual; atenção aos estímulos visuais e auditivos), 15 itens que avaliam o tônus muscular e seis itens que verificam os reflexos primitivos e profundos ${ }^{34}$.

Durante a aplicação do teste também são acompanhadas as seis categorias do estado comportamental ${ }^{35,36}$. Os RN são classificados, como proposto por Dubowitz e Dubowitz ${ }^{34}$, em normais, limítrofes ou anormais. Dubowitz e Dubowitz ${ }^{34}$ reavaliaram 116 bebês (27 a 34 semanas de gestação) com um ano de idade e, dos 62 bebês avaliados como neurologicamente normais no período neonatal, somente $35 \%$ se mostraram normais com um ano de idade e, das 39 crianças consideradas anormais no período neonatal, 35\% eram normais com um ano. O valor preditivo positivo foi de apenas $64 \%$ e o negativo de $92 \%{ }^{18}$.

\subsection{PEABODY DEVELOPMENTAL MOTOR SCALE (ESCALA PDMS)}

A escala PDMS foi desenvolvida por Folio e Fewell entre 1969 e 1982, sendo revi- sada e atualizada em 2000, dando origem a PDMS - segunda edição. O teste tem como objetivos: identificar lactentes com atraso no desenvolvimento motor e suas necessidades; avaliar o desenvolvimento motor ao longo do tempo ou em resposta a intervenção, bem como identificar os objetivos motores e as estratégias de intervenção ${ }^{37}$.

A PDMS apresenta uma grande amostra de dados normativos e é padronizada para mensurar habilidades motoras grossas e finas de crianças desde o nascimento até cinco anos de idade ${ }^{37}$. Tanto a escala motora grossa quanto a escala motora fina podem ser administradas em 45 a 60 minutos. A escala motora grossa contém 170 itens cujas tarefas são: reflexos, equilíbrio, atividades estáticas e de locomoção, a recepção e propulsão de objetos. A escala motora fina contém 112 itens. Sendo que as tarefas motoras finas incluídas nesta avaliação são: pressão, o uso da mão, a coordenação olho-mão e a destreza manual. As correlações de confiabilidade teste reteste e interobservador dessa escala são excelentes $^{36,38}$.

\subsection{TIMP - TEST OF INFANT MOTOR PERFORMANCE}

O TIMP (Test of Infant Motor Performance) é um teste de função motora do comportamento usado por profissionais da saúde que trabalham na intervenção precoce de RN. Foi idealizado por Campbell e colaboradores em 1993, para ser aplicado em crianças prétermo e a termo de 32 semanas pósconcepcionais até a idade de quatro meses, buscando avaliar sua qualidade de movimento, controle e alinhamento postural, equilíbrio e coordenação de acordo com sua evolução e habilidades funcionais ${ }^{39}$.

A criança pode ser avaliada estando ainda na UTI neonatal, desde que apresente condições clínicas estáveis, sem sinais de estres- 
se. O teste foi projetado inicialmente para ser realizado por fisioterapeutas e terapeutas ocupacionais que atuam diretamente com a motricidade e que tenham experiência com intervenção precoce em crianças de risco ${ }^{39}$.

A primeira versão do teste, proposta por Girolami e Campbell ${ }^{39}$, era composta de 43 itens, sendo criada para um estudo sobre a efetividade do tratamento em crianças prematuras de alto risco, em 1983. O instrumento foi desenvolvido a partir das teorias de aprendizado motor que ressaltavam a importância da estabilidade e a orientação de alinhamento no espaço, relacionadas à interação do ambiente e às modificações neuromotoras de auto-organização do indivíduo. A versão mais atual do teste foi desenvolvida com fotos ilustrativas, para ser também utilizado como uma ferramenta educacional para os pais em relação à evolução do $\mathrm{RN}^{40}$.

A avaliação é composta de 27 itens, pontuados com base na observação da atividade espontânea da criança em presente ou ausente, e mais 25 itens eliciados avaliados pelo examinador de acordo com um formato padronizado em uma escala de cinco ou seis pontos que descrevem comportamentos específicos a serem notados, variando de menos maduro a com resposta completa. Os itens do instrumento realizam uma avaliação direcionada para o desenvolvimento do controle de cabeça e controle seletivo dos membros, avaliando o repertório motor característico do primeiro trimestre de vida em variadas posições no espaço, com contribuição de estímulos visuais e auditivos ${ }^{40,41}$.

O teste foi validado a partir de uma amostra selecionada, mantendo proporções adequadas de sexo e raça para atender à diversidade da população estadunidense. A confiabilidade e sensibilidade foram verificadas na validação e evidenciaram excelentes resultados no terceiro mês. A confiabilidade interobservador é de 0,95 e no teste reteste de 0,89 . A sensibilidade é de 0,92 , porém a especificidade é de $0,76^{40,41}$.

\section{11 ALBERTA INFANT MOTOR SCALE (AIMS)}

A escala Alberta Infant Motor Scale (AIMS) foi construída e validada na Universidade de Alberta, no Canadá, especificamente no departamento de Medicina e Reabilitação por Piper e Darrah ${ }^{43}$, com o objetivo de avaliar o desenvolvimento motor grosso de crianças.

A Alberta Infant Motor Scale (AIMS) é uma escala que se propõe a avaliar e monitorar o desenvolvimento motor amplo de lactentes por meio da observação da atividade motora espontânea, desde o nascimento até os 18 meses de vida ou até a aquisição da marcha independente. Sua amostra normativa foi constituída de uma coorte de 2.202 lactentes, representativos de todas as crianças nascidas em Alberta, Canadá, entre março de 1990 e junho de $1992^{43}$.

Amplamente utilizada para documentar o desenvolvimento de aquisições motoras grossas e identificar atraso no desenvolvimento motor dos recém-nascidos a termo e pré-termos. Sendo indicada para acompanhar o desenvolvimento de crianças com suspeitas de atraso motor, bem como para avaliar a eficácia de programas de intervenção precoce ${ }^{43,44}$.

O instrumento criado por essas duas fisioterapeutas canadenses (AIMS) é considerado uma medida observacional do desempenho motor infantil que aborda conceitos do desenvolvimento motor como: neuromaturação, perspectiva da dinâmica motora e avaliação da sequência do desenvolvimento motor. Os padrões motores e as posturas são analisados usando-se três critérios: alinhamento postural, movimentos antigravitacionais e superfície de contato ${ }^{43,45}$.

AAIMS é um protocolo de avaliação que tem sido muito utilizado em vários estudos em nosso país, por ser considerado útil, prático e de baixo custo na avaliação do desenvolvimento neuropsicomotor de lactentes prematuros nos programas de seguimento na rede pública brasileira ${ }^{46}$. No entanto, faz-se necessário verifi- 
car suas propriedades psicométricas, pois níveis satisfatórios de validade e confiabilidade de um instrumento de avaliação não são garantidos quando ele é utilizado em uma população culturalmente diferente daquela para o qual fora desenvolvido ${ }^{47}$.

Muitos testes são utilizados para triagem e diagnóstico de anormalidades do desenvolvimento de prematuros, identificando-se, neste estudo, 11 testes, como sendo mais indicados na avaliação de prematuros.

Cada instrumento abordado na presente pesquisa apresenta vantagens e desvanta-

\section{REFERÊNCIAS}

1. Rugolo, LMSS. Growth and developmental outcomes of the extremely preterm infant. J Pediatr. 2005; 81(1): 101-110.

2. Formiga CKER, Pedrassani ES, Tudella E. Desenvolvimento motor de lactentes pré-termos participantes de um programa de intervenção fisioterapêutica precoce. Rev. bras. Fisioter. 2004; 8(3): 239-245

3. Linhares MBM, Carvalho AEV, Machado C, Martinez FE. Desenvolvimento de bebês nascidos pré-termo no primeiro ano de vida. Paidéia. 2003; 13(25): 59-72.

4. Obana AY, Oshiro M. A terapia ocupacional em bebês de risco: reflexos sobre a clínica. Cadernos do Centro Universitário São Camilo. São Paulo. jul/set 2002; 8(3): 58-61.

5. Almeida KM, Dutra MV, de Mello RR, Reis AB, Martins PS. Concurrent validity and reliability of the Alberta Infant Motor Scale in premature infants. J Pediatr (Rio J). 2008; 84(5): 442-448.

6. Santos DCC, Ravanini SG. Aspectos do diagnóstico do desenvolvimento motor. In: Moura MV, Gonçalves VM. Neurologia do desenvolvimento da criança. Rio de Janeiro: Revinter, 2006. p. 258-69. gens, sendo que o examinador deve escolher o exame mais adequado aos seus objetivos, seja na sua utilização em pesquisas, triagem clínica ou na verificação da eficiência da intervenção precoce proposta.

Além disso, devem estar atentos às propriedades psicométricas, pois bons escores de confiabilidade e validade são determinantes na eficácia do teste, principalmente quando aplicados em determinada faixa etária, já que alguns instrumentos são mais confiáveis e preditivos quando aplicados em determinadas idades ou condições clínicas.

7. Francisco RN, Caon G, Bissani C, Silva CA, Sousa M, Silva L. Características neuropsicomotoras de crianças de alto risco neurológico atendidas em um programa de follow up. Pediatria Moderna. 2006; 42(2): 79-85.

8. Mancini MC, Teixeira S, de Araújo LG, Paixão ML, Magalhães LC, Coelho ZAC, et al. Estudo do desenvolvimento da função motora aos 8 e a 2 meses de idade, em crianças pré-termo e atermo. Arq. Neuropsiquiatr. 2002; 60(4): 974-980.

9. Gesell, A, Amatruda CS. Diagnóstico do desenvolvimento: avaliação do desenvolvimento neuropsicológico no lactente e na criança pequena: o normal e o patológico. 4th ed. Rio de Janeiro: Atheneu; 2000.

10. Gesell, A, Ames LB. The development of handiness. J Genet Psychol 1947; 70: 155-75

11. Ferreira AT, Silva MMA, Silva L, Merighi LBM, Miranda AM, De-Vitto LPM, Lamônica DAC. Desempenho comunicativo em trigêmeos prematuros. Rev CEFAC, São Paulo. 2008; 10(1): 15-21.

12. Hage SRV, Joaquim RSS, Carvalho KG, Padovani CR, Guerreiro MM. Diagnóstico de crianças com alterações específicas de linguagem por meio de escala de de- 
senvolvimento. Arq Neuropsiquiatr. 2004; 62(3-A): 649-653.

13. Bayley N. Bayley Scales of Infant Development. San Antonio, TX: The Psychological Corporation, 1969.

14. Tecklin JS. Fisioterapia Pediátrica. 3 ed. Porto Alegre: Artmed, 2002.

15. Bayley, N. Bayley Scales of Infant Development - Second Edition, Administration Manual. San Antonio, TX: The Psychological Corporation, 1993.

16. Bayley, N. Bayley Scales of Infant and Toddler Development - Third Edition, Administration Manual. San Antonio, TX: The Psychological Corporation, 2006.

17. Lordelo ER, Chalhub AA, Guirra RC, Carvalho CS. Contexto e desenvolvimento cognitivo: freqüência à creche e evolução do desenvolvimento mental. Psicologia: Reflexão e Crítica. 2007; 20(2): 324-334.

18. Sweeney JK, Swanson MW. Crianças de baixo peso ao nascer: cuidados neonatais e acompanhamento. In: Umphred DA. Reabilitação neurológica. $4^{\mathrm{a}}$ ed. São Paulo: Manole; 2004. p. 213-71.

19. Frankenburg WK, Dodds JB. The Denver Developmental Screening Test. J Pediatr. 1967; 71(2): 181-191.

20. Rezende MA, Beteli VC, Santos JLF. Avaliação de habilidades de linguagem e pessoal-sociais pelo Teste de Denver II em instituições de educação infantil. Acta Paul Enferm. 2005; 18(1): 56-63.

21. Frankenberg WK, Dodds J, Archer P, Shapiro H, Bresnick B.The Denver II: a major revision and restandardization of Denver developmental screening test. Pediatrics 1992; 89-91.

22. Rezende MA, Beteli VC, Santos JLF. Follow-up of the child's motor abilities in day-care centers and pre-schools. Rev Latino-am Enfermagem. 2005 set-oct; 13(5): 619-25.

23. Souza SC, Leone C, Takano AO, Moratelli HB. Desenvolvimento de pré-escolares na educação infantil em Cuiabá, Mato Grosso, Brasil. Cad. Saúde Pública, Rio de Janeiro. 2008 aug; 24(8): 1917-1926.

24. Santos RS, Araújo APQC, Porto MAS. Early diagnosis of abnormal development of preterm newborns: assessment instruments. J Pediatr (Rio J). 2008; 84(4): 289-299.

25. Tecklin, JS. Fisioterapia Pediátrica. $3^{\mathrm{a}}$ ed. Porto Alegre: Artmed, 2002, 479 p.

26. Umphred DA. Reabilitação Neurológica. 4 ed. Barueri: Manole, 2004. p.475-505.

27. Effgen SK. Fisioterapia Pediátrica: atendendo as necessidades das crianças. Rio de Janeiro, Guanabara Koogan, 2007

28. Zdanska-Brincken M, Wolanski N. graphic method for evaluation of motor development in infants. Dev Med Child Neurol. 1969; 11(2): 228-41.

29. Brazelton TB, Nugent JK. Neonatal behavioral assessment scale. 3 ed. London: Mac Keith Press; 1995.

30. Harris SR, Brady DK. Infant neuromotor assessments instruments: a review. In: Sweeney JK. The high-risk neonate: developmental therapy perspectives. Binhhamton, NY: Haworth Press, 1986. p. 135-8.

31. Lacerda TTB, Magalhães LC. Análise da validade dos itens do Movement Assessment of Infants - MAI - para crianças pré-termo. Rev. Bras. Saúde Matern. Infant., Recife. Jul/Set 2006; 6(3): 297308.

32. Schirmer CR, Portuguez MW, Nunes ML. Clinical assessment of language development in children at age 3 years that were born preterm. Arq Neuropsiquiatr. 2006; 64(4): 926-931.

33. Salokorpi T, Rajantie I, Kivikko I, Haajanen R, Rajantie, J. Predicting neurological disorders in infants with extremely low birth weight using the movement assessment of infants. Pediatr Phys Ther. 2001; 13(3): 106-109. 
34. Dubowitz LMS, Dubowitz V. The neurological assessment of the preterm and full term newborn infant. Clinics in Development Medicine, 79. London: SIMP; 1981.

35. Barradas J, Fonseca A, Guimarães CL, Lima GM. Relationship between positioning of premature infants in Kangaroo Mother Care and early neuromotor development. J Pediatr (Rio J). 2006; 82: 475-80.

36. Mello RR, Dutra MVP, Silva KS, Lopes JMA. Valores de predição da avaliação neurológica e ultra-sonográfica cerebral neonatal em relação ao desenvolvimento de prematuros de muito baixo peso. Rev Saúde Púb. 1998; 32(5): 420-429.

37. Folio R, Fewel R. The Peabody Developmental Motor Scales (Manual). Astin: Pro Ed, 1983.

38. Ponjaert-Kristoffersen I, Tjus T, Nekkebroeck J, Squires J, Verté D, Heimann M, et al. Psychological followup study of 5-year-old ICSI children. Hum Reprod. 2004; 19(12): 2791-2797.

39. Campbell SK, Girolami GL, Kolobe THA, Osten ET, Lenke MC. Test of infant motor performance. 3 ed. Chicago: [s/ed]; 2001.

40. Campbell SK, Kolobe TH, Osten ET, Lenke M, Girolami GL. Construct validity of infant motor performance. Pediatr Phys Ther. 1995; 75: 585-586.
41. Campbell SK. Test-retest reliability of the test of infant motor performance. Pediatr Phys Ther. 1999; 11: 60-66.

42. Santos RS, Araújo AP, Porto MA. Early diagnosis of abnormal development of preterm newborns: assessment instruments. J Pediatr.(Rio J). 2008; 84(4): 289-299.

43. Piper MC, Darrah JM. Motor Assessment of the Developing Infant. Alberta: WB Saunders, 1994.

44. Piper MC, Pinnell LE, Darrah J, Maguire T, Byrne PJ. Construction and validation of the Alberta Infant Motor Scale (AIMS). Can J Public Health. 1992; 83(2): 546-550.

45. Bartlett DJ, Fanning JE. Use of the Alberta Infant Motor Scale to characterize the motor development of infants born preterm at eight months corrected age. Phys Occup Ther Pediatr. 2003; 23(4): 31-45.

46. Manacero S, Nunes ML. Evaluation of motor performance of preterm newborns during the first months of life using the Alberta Infant Motor Scale (AIMS). J Pediatr (Rio J). 2008; 84(1): 53-59.

47. Vieira MEB, Ribeiro FV, Formiga CKMR. Principais instrumentos de avaliação do desenvolvimento da criança de zero a dois anos de idade. Revista Movimenta. 2009; 2(01): 23-31.

Recebido em: 26/mai./10 Modificado em: 30/ago./10 Aceito em: 10/dez./10 